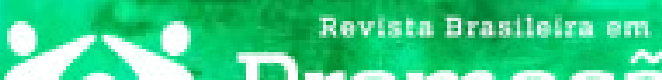

\section{DESAFIOS DO FINANCIAMENTO DA ATENÇÃO PRIMÁRIA À SAÚDE: REVISÃo INTEGRATIVA}

\section{Challenges of primary health care financing: integrative review \\ Desafios de la financiación de la atención primaria de salud: revisión integrativa}

\author{
Ivanise Brito da Silva \\ Universidade Federal do Pernambuco - UFPE - Recife (PE) - Brasil.
}

\section{RESUMO}

Objetivo: Avaliar como ocorre o financiamento da Atenção Primária à Saúde (APS) no Brasil, refletindo sobre os avanços e entraves existentes. Métodos: Trata-se de uma revisão integrativa realizada no período de junho e julho de 2016. A busca dos estudos foi realizada nas bases de dados eletrônicas Centro Latino-Americano e do Caribe de Informação em Ciências da Saúde (BIREME) e na Biblioteca Virtual Scientific Eletronic Library Online (SciELO), utilizando-se como descritores de assunto "primary health care" e "financing". Os critérios de elegibilidade do estudo foram artigos científicos brasileiros, indexados no período de 1994 a 2016, escritos nos idiomas inglês, português ou espanhol, e que estivessem disponíveis na íntegra. Foram excluídas as publicações no formato de tese, dissertação, capítulo de livro e as que não tinham relação com o tema da pesquisa, bem como as que tratavam de estudos sobre financiamento APS realizados fora do Brasil. Resultados: Foram encontradas 290 publicações. Após aplicação dos critérios de inclusão e exclusão, obteve-se 15 artigos dos quais emergiram duas categorias temáticas: "Subfinanciamento: um desafio para a APS" e "Descentralização e os entraves no financiamento da APS". Conclusão: Os achados desta revisão identificaram diferentes entraves no financiamento e no estabelecimento de políticas de fortalecimento da atenção primária do país, evidenciando que os repasses e alocações dos recursos devem ser analisados de forma mais criteriosa e reflexiva. Os artigos avaliados apresentam como principais problemas enfrentados no financiamento: verbas insuficientes, necessidade de serem estabelecidos parâmetros mais claros para os repasses intergovernamentais, criação de uma gestão que entenda e dialogue com as dificuldades locais, e maior autonomia dos municípios na definição de prioridades em contrapartida aos financiamentos destinados ao custeio de programas específicos.

Descritores: Atenção Primária à Saúde; Financiamento; SUS.

\section{ABSTRACT}

Objective: To evaluate the way Primary Health Care financing occurs in Brazil, reflecting on the existing advances and obstacles. Methods: This is an integrative review carried out in the period of June and July 2016. The search was conducted in the electronic databases Latin American and Caribbean Center on Health Sciences Information (BIREME) database and Scientific Electronic Library Online (SciELO), using as descriptors "primary health care" and "financing". The eligibility criteria of the study were Brazilian scientific articles, indexed in the period from 1994 to 2016, written in English, Portuguese or Spanish, and being available in full text. Publications in the thesis, dissertation and book chapter format, those not related to the research topic, as well as those dealing with studies on PHC financing carried out outside Brazil, were excluded. Results: The search resulted in 290 publications. After applying the inclusion and exclusion criteria, 15 articles were obtained, from which two thematic categories emerged: "Underfinancing: a challenge for PHC" and "Decentralization and obstacles to PHC financing". Conclusion: The findings of this review identified different obstacles to the financing and establishment of policies for the improvement of primary health care in the country, pointing out that transfers and allocations of resources should be analyzed in a more discerning and reflective manner. The articles examined present as the main problems faced in financing: insufficient funds, the need to establish clearer parameters for intergovernmental transfers, the development of a management that understands and interact with the local difficulties, and the greater autonomy of the municipalities in setting priorities in counterpart to financing directed at specific programs.

Descriptors: Primary Health Care; Financing; Unified Health System.

\section{RESUMEN}

Objetivo: Evaluar cómo se da la financiación de la Atención Primaria de Salud (APS) en Brasil con una reflexión sobre los avances y las trabas existentes. Métodos: Se trata de una revisión integrativa realizada entre junio y julio de 2016. La búsqueda de los estudios fue realizada en las bases de datos electrónicas: Centro Latino-Americano y del Caribe de Información e Ciencias de la Salud (BIREME) 
y en la Biblioteca Virtual Scientific Eletronic Library Online (SciELO) utilizándose los descriptores de asunto "primary health care" y "financing". Los criterios de elegibilidad del estudio fueron artículos cientificos brasileños indexados en el periodo de $1994-2016$ escritos en los idiomas inglés, portugués o español y que estuvieran disponibles en texto completo. Fueron excluidas las publicaciones en el formato de tesis, los trabajos de fin de grado, los capitulos de libros y las que no tenían relación con el tema investigado así como los estudios sobre la financiación del APS realizados fuera de Brasil. Resultados: Se encontraron 290 publicaciones. Después de la aplicación de los criterios de inclusión y exclusión se quedaron 15 artículos de los cuales emergieron dos categorías de temas: "Subfinanciación: un desafio para la APS" y "Descentralización y las trabas para la financiación de la APS". Conclusión: Los hallazgos de esa revisión identificaron distintas trabas para la financiación y el establecimiento de políticas de fortalecimiento de la atención primaria del país evidenciando que los repases y los destinos de los recursos deben ser analizados con más criterios y reflexiones. Los artículos evaluados presentan los siguientes problemas principales afrontados para la financiación: las verbas insuficientes, la necesidad de parámetros más claros para los repases intergubernamentales, la creación de una gestión que comprenda y dialogue con las dificultades locales, una mayor autonomía de los municipios para la definición de prioridades en contrapartida para las financiaciones para el costeo de programas especificos.

Descriptores: Atención Primaria de Salud; Financiación del Capital; Sistema Único de Salud.

\section{INTRODUÇÃO}

O Sistema Único de Saúde (SUS) criado em 1988, fruto da reforma sanitária, foi homologado pela Constituição de 1988, trazendo como princípios a universalidade, a equidade e a integralidade. Além disso, preconizou a participação popular e a descentralização. Para regulamentar seus princípios e diretrizes, foram sancionadas, em 1990, as Leis n ${ }^{\circ} 8080 / 90$ e n $n^{\circ} 8182 / 90^{(1)}$.

A descentralização promoveu maior participação política dos municípios na tomada de decisão sobre as prioridades da saúde local e favoreceu novos mecanismos de financiamento e transferência de responsabilidades pela execução direta de serviços de saúde, principalmente os da atenção primária ${ }^{(2)}$. Com a descentralização e as mudanças do modelo de gestão, os municípios ganharam mais autonomia e responsabilidade sobre os atendimentos da baixa complexidade.

A Atenção Primária à Saúde (APS) é vista como primeiro contato para o cuidado, sendo porta de entrada para os usuários do SUS. Com base num atendimento comunitário, em detrimento ao individual e unicausal, visa realizar ações que promovam o cuidado integral, respondendo, dessa forma, a maior parte das necessidades de saúde de uma população. Para isso, precisa ser articulada e direcionada por políticas amplas ${ }^{(3)}$.

A Norma Operacional Básica de 1996 (NOB 96) estimula o processo de descentralização e estabelece o Programa Saúde da Família (PSF) como parte de um conjunto de iniciativas que fortalece a APS ${ }^{(4)}$. Segundo a NOB, o Piso Assistencial Básico (PAB), para financiar procedimentos ambulatoriais e incentivar programas como PSF e Agentes Comunitários de Saúde $(\mathrm{ACS})^{(5)}$, deve ser calculado com base no tamanho da população local.

A vinculação de recursos à saúde contribui para a ampliação gradual do gasto até o percentual mínimo estabelecido. Os recursos federais são definidos pelo tamanho da população e tipo de gestão municipal, além disso, há recursos adicionais destinados a programas específicos ${ }^{(6)}$. A Emenda Constitucional 29 (EC 29) estabelece que a União investirá do seu orçamento $5 \%$ do ano anterior mais a correção do PIB nominal. Para estados e municípios, a quota seria $12 \%$ e $15 \%$ respectivamente ${ }^{(7)}$, diminuindo as desigualdades no financiamento da saúde ao equalizar o percentual mínimo de cada ente federativo ${ }^{(6)}$.

Em 2011, foi aprovada a Política Nacional de Atenção Básica (PNAB) pela Portaria $n^{\circ}$ 2.488/2011, com objetivo de reorganizar o sistema de saúde a partir da atenção primária por meio de um modelo horizontal baseado em rede de atenção à saúde. A reorientação dos setores de saúde utilizando a APS como componente fundamental cria um sistema conduzido por ações sanitárias mais eficazes e eficientes, objetivando fazer cumprir o que é preconizado pela lei ${ }^{(8)}$.

Apesar de a PNAB ter sido aprovada apenas em 2011, desde a década de 90 o Brasil vem passando por mudanças significativas na forma de direcionar os cuidados da atenção primária. O PSF surgiu em 1994 e passou a ser encarado pelo Ministério da Saúde como a principal estratégia de organização da atenção básica à saúde no país ${ }^{(9)}$.

Considerando a importância da APS para a reorganização dos serviços de saúde do país e todos os avanços e desafios inerentes a esse novo "modelo" de saúde, buscou-se, neste artigo, avaliar como ocorre o financiamento da Atenção Primária à Saúde (APS) no Brasil, refletindo sobre os avanços e entraves existentes.

\section{MÉTODOS}

Trata-se de pesquisa bibliográfica, do tipo revisão integrativa, método que promove a síntese dos resultados de pesquisa ${ }^{(10)}$. Este estudo foi composto por seis etapas: identificação do tema ou questionamento da revisão integrativa; amostragem ou busca na literatura; categorização dos estudos; avaliação dos estudos incluídos na revisão; interpretação dos resultados; e síntese do conhecimento evidenciado nos artigos analisados ou apresentação da revisão integrativa ${ }^{(11)}$. 
O estudo ocorreu nos meses de junho e julho de 2016, tendo como pergunta de partida: Como acontece o financiamento da atenção primária no Brasil? A coleta de artigos foi realizada nas bases de dados Centro Latino-Americano e do Caribe de Informação em Ciências da Saúde (BIREME) e na Biblioteca Virtual Scientific Eletronic Library Online (SCIELO), utilizando-se como descritores de assunto "primary health care" e "financing", utilizando-se o booleano "and". Os critérios de elegibilidade do estudo compreenderam artigos científicos brasileiros, indexados no período de 1994 a 2016 , escritos nos idiomas inglês, português ou espanhol, e que estivessem disponíveis na íntegra. Excluíram-se as publicações no formato de tese, dissertação, capítulo de livro e as que não tinham relação com o tema da pesquisa, bem como, as que tratavam de estudos sobre financiamento APS realizados fora do Brasil.

A análise dos trabalhos selecionados foi realizada com um instrumento que apresenta objetivos, resultados e conclusão de cada estudo. Realizou-se um fichamento das publicações selecionadas, objetivando identificar a forma de financiamento da atenção primária no Brasil, bem como os avanços e desafios. Realizaram-se leituras críticas com o objetivo de responder a questão condutora, obtendo-se a categorização temática de acordo com os conteúdos evidenciados.

\section{RESULTADOS}

Foram encontrados 290 artigos na busca inicial, entretanto, somente 15 estavam de acordo com os critérios de elegibilidade do presente estudo, sendo 5 oriundos da base de dados Scielo e 10 da Bireme.

Os principais resultados dos estudos encontrados foram apresentados em um quadro para melhor visualização das evidências (Quadro I). Quanto aos objetivos propostos pelas publicações, foram identificados trabalhos que avaliaram o financiamento APS, as leis que regulamentam a atenção primária, o impacto do financiamento ao longo do tempo e a participação dos entes federativos no financiamento.

Quadro I - Características dos artigos analisados descritas por autor, ano de publicação, objetivo e conclusão dos estudos.

\begin{tabular}{|c|c|c|c|}
\hline Autor & Ano & Objetivo & Conclusão \\
\hline Castro; Machado ${ }^{(2)}$ & 2010 & $\begin{array}{l}\text { Analisar a condução federal da } \\
\text { política de atenção primária à saúde } \\
\text { no Brasil de } 2003 \text { a } 2008 \text {. }\end{array}$ & $\begin{array}{l}\text { No que concerne ao financiamento, houve discreto } \\
\text { aumento da participação da atenção básica no } \\
\text { orçamento federal, reajustes e criação de novos } \\
\text { incentivos, alguns visando à equidade. }\end{array}$ \\
\hline Marques; Mendes ${ }^{(4)}$ & 2002 & $\begin{array}{l}\text { Analisar a evolução do gasto e do } \\
\text { financiamento da atenção à saúde no } \\
\text { país, as prioridades e as estratégias } \\
\text { de financiamento e relacionar a } \\
\text { Norma Operacional Básica de } 1996 \\
\text { com a política de transferência de } \\
\text { recursos para os municípios, adotada } \\
\text { pelo governo federal. }\end{array}$ & $\begin{array}{l}\text { A política da descentralização incentiva a despesa } \\
\text { em determinados programas e impede que os } \\
\text { municípios definam livremente sua política de } \\
\text { saúde, introduzindo o paradoxo da existência da } \\
\text { "pobreza" em um quadro de recursos "abundantes" } \\
\text { e garantidos pelos incentivos. }\end{array}$ \\
\hline Mendes et al. ${ }^{(5)}$ & 2011 & $\begin{array}{l}\text { Aplicar uma metodologia de } \\
\text { alocação equitativa dos recursos } \\
\text { federais do SUS para os estados } \\
\text { e municípios brasileiros, tanto } \\
\text { para procedimentos da atenção } \\
\text { básica como para média e alta } \\
\text { complexidade. }\end{array}$ & $\begin{array}{l}\text { Partindo de um valor per capita base de aproxi- } \\
\text { madamente } \mathrm{R} \$ 27,00 \text {, referente a recursos federais } \\
\text { repassados aos municípios para financiar procedi- } \\
\text { mentos da atenção básica, a metodologia proposta } \\
\text { conduziu à correção desse valor per capita pelo } \\
\text { Indicador de Necessidades Socioeconômica e } \\
\text { Sanitária (INSES). }\end{array}$ \\
\hline Vazquez $^{(6)}$ & 2011 & $\begin{array}{l}\text { Analisar os impactos da vinculação } \\
\text { de receitas e das transferências } \\
\text { condicionadas à oferta sobre o } \\
\text { financiamento da saúde. }\end{array}$ & $\begin{array}{l}\text { Há complementaridade entre vinculação de } \\
\text { receitas e transferências condicionadas à oferta } \\
\text { que aumentou a participação dos governos } \\
\text { subnacionais no financiamento da saúde e forneceu } \\
\text { incentivos para a descentralização da atenção } \\
\text { básica, segundo diretrizes definidas centralmente, } \\
\text { e reduziu as desigualdades em relação ao gasto } \\
\text { per capita em saúde dos municípios. }\end{array}$ \\
\hline
\end{tabular}




\begin{tabular}{|c|c|c|c|}
\hline Santos et al. ${ }^{(12)}$ & 2015 & $\begin{array}{l}\text { Analisar o papel do estado de } \\
\text { Pernambuco quanto às prioridades } \\
\text { de investimento nos níveis de } \\
\text { complexidade dos serviços e no } \\
\text { processo de regionalização com } \\
\text { base na visão dos diversos atores da } \\
\text { política de saúde no estado. }\end{array}$ & $\begin{array}{l}\text { Falta de empenho do estado em financiar ações da } \\
\text { atenção básica, contudo reconhecem o avanço no } \\
\text { processo de regionalização. }\end{array}$ \\
\hline Lima; Andrade ${ }^{(16)}$ & 2009 & $\begin{array}{l}\text { O artigo analisa as condições de } \\
\text { financiamento do Sistema Único de } \\
\text { Saúde nos municípios brasileiros } \\
\text { com mais de } 100 \text { mil habitantes. }\end{array}$ & $\begin{array}{l}\text { Verificaram-se perfis variados de receitas } \\
\text { municipais nas diversas regiões e estados } \\
\text { do Brasil e distintos graus de dependência } \\
\text { orçamentária dos municípios às principais fontes } \\
\text { de recursos vinculados. Embora a diversidade de } \\
\text { fontes indique múltiplos caminhos para obtenção } \\
\text { de recursos, o estudo sugere alguns entraves para } \\
\text { o financiamento da saúde nos grandes municípios. }\end{array}$ \\
\hline Rosa; Coelho ${ }^{(17)}$ & 2011 & $\begin{array}{l}\text { Identificar o fluxo das contas } \\
\text { municipais desde as fontes de } \\
\text { financiamento até as atividades de } \\
\text { saúde e problematizar e avaliar a } \\
\text { alocação dos recursos financeiros e a } \\
\text { execução orçamentária do Programa } \\
\text { de Saúde da Família no município } \\
\text { de Santo Antônio de Jesus. }\end{array}$ & $\begin{array}{l}\text { O resultado da pesquisa revelou gastos per } \\
\text { capita das } 16 \text { unidades do PSF, que variaram de } \\
\mathrm{R} \$ 465,40 \text { a R } \$ 62,3 \text { per capita/unidade/ano. O } \\
\text { gasto médio de uma unidade do PSF foi de } \$ \$ \\
17.302 / \text { unidade/mês em } 2005 \text {, e o financiamento } \\
\text { do governo federal foi R } \$ 2.834 / \text { unidade/mês. } \\
\text { Sendo assim um financiamento irrisório do PSF } \\
\text { por parte do governo federal, uma inexistência } \\
\text { de cofinanciamento estadual e grande esforço do } \\
\text { município em ampliar o acesso. }\end{array}$ \\
\hline Scatena; Tanaka ${ }^{(18)}$ & 2000 & $\begin{array}{l}\text { Analisar o financiamento do Sistema } \\
\text { Único de Saúde no estado de Mato } \\
\text { Grosso, buscando identificar o } \\
\text { modelo assistencial que vem se } \\
\text { conformando a partir de } 1994 .\end{array}$ & $\begin{array}{l}\text { Não há perspectiva de incremento nos recursos } \\
\text { da saúde e o modelo atual está gerando redução } \\
\text { no volume das atividades de atenção primária, } \\
\text { resultando em maior demanda ao segmento de } \\
\text { média e alta complexidade. }\end{array}$ \\
\hline Campos $^{(19)}$ & 2012 & $\begin{array}{l}\text { Resgatar significados do movimento } \\
\text { da reforma sanitária e do movimento } \\
\text { municipal de saúde no contexto das } \\
\text { décadas de } 70 \text { e } 80 \text {, sua força social, } \\
\text { política e inovadora na reconstrução } \\
\text { democrática na época. }\end{array}$ & $\begin{array}{l}\text { Existem desigualdades nos níveis de atenção e } \\
\text { os repasses federais aos estados e municípios } \\
\text { são ainda fragmentados por programa e projeto } \\
\text { federal, e não globais, segundo as metas do } \\
\text { planejamento municipal, regional e estadual, o } \\
\text { que mantém o modelo convencional, e não das } \\
\text { relações constitucionais. }\end{array}$ \\
\hline Heimann et al. ${ }^{(20)}$ & 2011 & $\begin{array}{l}\text { Analisar a atenção primária em } \\
\text { saúde como estratégia para alcançar } \\
\text { sistemas integrais e universais. }\end{array}$ & $\begin{array}{l}\text { Apesar da implantação do Piso assistencial } \\
\text { básico, Emenda constitucional no } 29 \text {, os novos } \\
\text { mecanismos de financiamento do gestor federal } \\
\text { ainda são baixos; o financiamento público e os } \\
\text { recursos são insuficientes. Além disso, a atenção } \\
\text { primária não dispõe de orçamento específico para } \\
\text { suas ações de implementação e execução. }\end{array}$ \\
\hline Domingos et al. ${ }^{(21)}$ & 2016 & $\begin{array}{l}\text { Analisar as normas jurídicas } \\
\text { fortalecedoras da atenção básica, } \\
\text { utilizando análise documental } \\
\text { referente às ações, programas e } \\
\text { estratégias de fortalecimento da } \\
\text { atenção primária priorizados pelo } \\
\text { Ministério da Saúde. }\end{array}$ & $\begin{array}{l}\text { O tema financiamento, relacionado a incentivos } \\
\text { e transferências de recursos, ocupou um lugar } \\
\text { central nas normas publicadas, principalmente } \\
\text { com relação às complementares. Tal situação } \\
\text { leva a uma reflexão sobre o financiamento } \\
\text { como instrumento de controle federal sobre os } \\
\text { municípios, pois estes têm de cumprir critérios } \\
\text { previstos nas normas para receber os recursos } \\
\text { necessários ao desenvolvimento das ações de } \\
\text { saúde. }\end{array}$ \\
\hline
\end{tabular}




\begin{tabular}{|c|c|c|c|}
\hline Sousa $^{(22)}$ & 2008 & $\begin{array}{l}\text { Apresentar dados de uma pesquisa } \\
\text { sobre a implantação do Programa } \\
\text { de Saúde da Família, realizada nos } \\
12 \text { municípios pioneiros, no que se } \\
\text { refere ao acesso aos serviços básicos } \\
\text { de saúde. }\end{array}$ & $\begin{array}{l}\text { No que se refere ao acesso aos serviços básicos } \\
\text { de saúde, o Programa de Saúde da Família } \\
\text { objetiva a diminuição das iniquidades, entretanto } \\
\text { enfrenta uma série de desafios de ordem social, } \\
\text { política, econômica, institucional e cultural, } \\
\text { materializados nas deficiências de coordenação, } \\
\text { gestão, financiamento e, sobretudo, nas práticas } \\
\text { assistenciais. }\end{array}$ \\
\hline Porto et al. ${ }^{(23)}$ & 2006 & $\begin{array}{l}\text { Analisar, a partir de microdados de } \\
1998 \text { e } 2003 \text {, a utilização de serviços } \\
\text { de saúde sob a perspectiva de seu } \\
\text { financiamento. }\end{array}$ & $\begin{array}{l}\text { O SUS é o principal financiador dos dois níveis } \\
\text { extremos de complexidade da atenção à saúde: o } \\
\text { de atenção básica e o de alta complexidade. }\end{array}$ \\
\hline Portela, Ribeiro ${ }^{(24)}$ & 2011 & $\begin{array}{l}\text { Analisar a estrutura de financiamento } \\
\text { setorial dos municípios de grande } \\
\text { porte por série histórica e sua relação } \\
\text { com a cobertura da Estratégia Saúde } \\
\text { da Família. }\end{array}$ & $\begin{array}{l}\text { Maior vulnerabilidade e dependência de } \\
\text { transferências federativas para os municípios de } \\
\text { menor porte e em áreas menos desenvolvidas. }\end{array}$ \\
\hline $\begin{array}{l}\text { Almeida; } \\
\text { Giovanella }^{(25)}\end{array}$ & 2008 & $\begin{array}{l}\text { Analisar as pesquisas de } \\
\text { monitoramento e avaliação em } \\
\text { Atenção Básica à Saúde, realizadas } \\
\text { e/ou financiadas pelo Ministério da } \\
\text { Saúde, e concluídas e publicadas } \\
\text { entre } 2000 \text { e } 2006 \text {. }\end{array}$ & $\begin{array}{l}\text { Entre as principais barreiras identificadas para } \\
\text { expansão destacaram-se o modelo de transferência } \\
\text { de recursos financeiros em função da faixa de } \\
\text { cobertura, a convivência paralela de diferentes } \\
\text { modelos de atenção básica, a ausência de } \\
\text { mecanismos de integração de rede, entre outras. }\end{array}$ \\
\hline
\end{tabular}

\section{DISCUSSÃO}

\section{Subfinanciamento: um desafio para a APS}

Um estudo ${ }^{(12)}$ aponta que, em 1980, o governo federal participava com 75\% do financiamento público na saúde, e os estados e municípios com 25\%. Em 1990, foi aprovada a Lei n ${ }^{\circ} 8142$, que dispõe sobre as transferências intergovernamentais de recursos financeiros da área de saúde, estabelecendo que a União deve participar com pelo menos 70\%. Entretanto, apesar da menor responsabilização da União com o financiamento da saúde, a década de 90 apresentou mudanças consideráveis na política nacional de atenção primária à saúde com o objetivo de ampliar o acesso ${ }^{(2)}$. As mudanças no arranjo federativo instaurado após a Constituição de 1988 e o processo de descentralização e municipalização foram importantes para a mudança do modelo hospitalocêntrico por um modelo de saúde preventiva ${ }^{(13)}$.

Uma pesquisa comparou os gastos públicos no Brasil e em outros países, sendo verificado que os gastos públicos em saúde no Brasil são muito baixos quando comparados com outros países, em dólares americanos, com paridade de poder de compra ${ }^{(14)}$. Já outro estudo ${ }^{(15)}$ apresentou a evolução dos valores per capita referentes às transferências federais da atenção básica, média e alta complexidade, ajustados pela inflação no período compreendido entre 1998 a 2006, e os autores destacaram que a atenção básica manteve um padrão de progressivo incremento entre 1998 e 2001 e a partir de 2003 . No entanto, embora com oscilações ao longo dos anos, as transferências de média e alta complexidade apresentaram valores per capita muito superiores em todo o período, e crescimento bem ascendente entre 2002 e 2004, registrando aumento da distância entre as curvas.

\section{A descentralização e os entraves no financiamento da APS}

O Ministério da Saúde, desde o início de 2000, tem priorizado municípios brasileiros com mais de 100 mil habitantes, com o objetivo de expandir e consolidar a atenção básica, já que tem sido evidenciada nesses centros urbanos uma baixa cobertura do Programa de Saúde da Família (PSF) ${ }^{(16)}$.

Mesmo antes da criação do SUS, o papel decisivo de alguns municípios já existia. Um exemplo disso está na atuação dos municípios no combate à poliomielite e ao sarampo, sendo evidenciado que, apesar dos resultados efetivos da descentralização, o governo federal, nos anos 80, iniciou a retração da sua participação no financiamento da saúde perante o crescimento da participação estadual e principalmente municipal ${ }^{(12)}$.

As relações fiscais e orçamentárias que se estabelecem entre os governos na federação brasileira são ineficazes do ponto de vista da redução das desigualdades entre municípios com grande porte populacional situados em distintas regiões e estados 
do país. As arrecadações municipais vinculadas à saúde das regiões Norte e Nordeste permanecem bem aquém das observadas na região Sul do país ${ }^{(1)}$. A descentralização, regionalização e municipalização aparecem como forma de tornar a saúde mais próxima das necessidades locais, contudo nem todos os municípios brasileiros apresentam condições política e financeira para suprir suas necessidades de saúde, mesmo quando se fala de serviços que demandam baixos investimentos tecnológicos.

Uma pesquisa realizada em um município da Bahia em 2005 revelou os gastos médios para a manutenção de um PSF, sendo identificados os gastos per capita de 16 unidades do PSF estudadas, que variaram de R \$62,30 a R \$ 465,40 per capita/ unidade/ano. Em 2005, o investimento federal foi de R \$2.834/unidade/mês. Os autores concluíram que não há cofinanciamento estadual, o que faz com que o município faça um grande esforço para ampliar o acesso aos serviços de saúde através da ESF ${ }^{(17)}$.

Uma pesquisa realizada no estado do Mato Grosso evidenciou que, do ponto de vista financeiro, os municípios matogrossenses ainda dependem grandemente dos recursos vindos de outras instâncias intergovermentais, sendo essa dependência maior entre os municípios com menos de 20.000 habitantes, mas também sendo significativa nos municípios de médio e grande porte $^{(18)}$. As pesquisas apontam a existência de perfis variados de receitas municipais nas diversas regiões e estados do Brasil, assim como distintos graus de dependência orçamentária dos municípios das principais fontes de recursos vinculados. Essas diferenças precisam ser trabalhadas para que se consiga alcançar uma atenção primária eficiente em todo território brasileiro.

As políticas descentralizadoras representam desafios para a gestão, tanto pela assunção de novas responsabilidades como para instituição de mudanças. Um estudo ${ }^{(19)}$ objetivou a criação de mecanismos organizacionais que tornem a responsabilidade sanitária dos entes federados, dos serviços e dos profissionais de saúde mais precisa e definida, visando à melhoria do serviço e à adoção de práticas humanizadas.

\section{CONCLUSÃO}

Os achados desta revisão identificaram diferentes entraves no financiamento e estabelecimento de políticas de fortalecimento da atenção primária do país, evidenciando que os repasses e a alocação dos recursos devem ser analisados de forma mais criteriosa e reflexiva. Os artigos avaliados apresentam como principais problemas enfrentados no financiamento: insuficiência de verba, necessidade de serem estabelecidos parâmetros mais claros para os repasses intergovernamentais, criação de uma gestão que entenda e dialogue com as dificuldades locais e maior autonomia dos municípios na definição de prioridades em contrapartida aos financiamentos destinados ao custeio de programas específicos.

Foram apontados também como dificuldades a escassez de recursos para a saúde e o modelo de assistência adotado, que transfere recursos da atenção primária à saúde para o segmento de atenção de maior complexidade. Nessa conjuntura, o financiamento da Atenção Primária à Saúde apresenta inviabilidades, como também há desafios relacionados à gestão do trabalho e à necessidade de novo aparato jurídico-institucional para a gestão regional.

\section{REFERÊNCIAS}

1. Scatena JHG, Tanaka OY. Utilização do Sistema de Informações hospitalares (SIH-SUS) e do Sistema de Informações Ambulatoriais (SIA-SUS) na Análise da Descentralização da Saúde em Mato Grosso. Inf Epidemiol SUS [Internet]. 2001 [acesso em 2016 Jul 25 ];10(1):19-30. Disponível em: http://scielo.iec.pa.gov.br/scielo.php?script=sci_arttext\&pid $=\mathrm{S} 0104-16732001000100003$

2. Castro ALB, Machado CV. A política de atenção primária à saúde no Brasil: notas sobre a regulação e o financiamento federal. Cad Saúde Pública [Internet]. 2010 [acesso em 2016 Maio 15];26(4):693-705. Disponível em: http://www.scielo. br/scielo.php?script=sci_arttext\&pid=S0102-311X2010000400012

3. Oliveira MAC, Pereira IC. Atributos essenciais da Atenção Primária e a Estratégia Saúde da Família. Rev Bras Enferm [Internet]. 2013 [acesso em 2016 Ago 26];66(1):58-64. Disponível em: http://www.scielo.br/scielo.php?script=sci_arttext \&pid=S0034-71672013000700020

4. Marques RM, Mendes A. política de incentivos do Ministério da Saúde para a atenção básica: uma ameaça à autonomia dos gestores municipais e ao princípio da integralidade? Cad Saúde Pública [Internet]. 2002 [acesso em 2016 Set 15];18(Supl):16371. Disponível em: http://www.scielosp.org/scielo.php?script=sci_arttext\&pid=S0102-311X2002000700016

5. Mendes A, Leite, MG, Marques RS. Discutindo uma metodologia para a alocação equitativa de recursos federais para o Sistema Único de Saúde. Saúde Soc [Internet]. 2011 [acesso em 2016 Ago 26];20(3):673-90. Disponível em: http://www. scielo.br/scielo.php?script=sci_arttext\&pid=S0104-12902011000300013

6. Vazquez DA. Efeitos da regulação federal sobre o financiamento da saúde. Cad Saúde Pública [Internet]. 2011 [acesso em 2016 Jun 02];27(6):1201-12. Disponível em: http:/www.scielo.br/scielo.php?script=sci_arttext\&pid=S0102$311 \times 2011000600017$ 
7. Brasil. Emenda Constitucional no 29. Ministério da Saúde [acesso em 2016 Jun 10]. Disponível em: http://conselho.saude. gov.br/web_sus20anos/20anossus/legislacao/emendaconstitucionaln29.pdf

8. Bursztyn I, Kushnir R, Giovanella L, Stolkiner A, Sterman-Heimann L, Riveros MI, et al. Notas para el estudio de la atención primaria en contextos de sistemas de salud segmentados. Rev Salud Pública [Internet]. 2010 [acesso em 2016 Jun 20];12(1):77-88. Disponível em: http://www.scielosp.org/pdf/rsap/v12s1/v12s1a06.pdf

9. Elias PE. Atenção Básica em Saúde: comparação entre PSF e UBS por estrato de exclusão social no município de São Paulo. Ciênc Saúde Coletiva [Internet]. 2006 [acesso em 2016 Mar 15];11(3):633-41. Disponível em: http://www.scielo. br/scielo.php?script $=$ sci_arttext\&pid=S1413-81232006000300012

10. Souza MT, Silva MD, Carvalho R. Revisão integrativa: o que é e como fazer. Einstein [Internet]. 2003 [acesso em 2016 Set 12];2010(8):102-6. Disponível em: http://www.scielo.br/pdf/eins/v8n1/pt_1679-4508-eins-8-1-0102.pdf

11. Mendes KDS, Silveira RCCP, Galvão CM. Revisão integrativa: método de pesquisa para a incorporação de evidências na saúde e na enfermagem. Texto \& Contexto Enferm [Internet]. 2008 [acesso em 2016 Out 12];17(4):758-64. Disponível em: http://www.scielo.br/pdf/tce/v17n4/18.pdf

12. Santos NR. SUS, política pública de Estado: seu desenvolvimento instituído e instituinte e a busca de saídas. Ciênc Saúde Coletiva [Internet]. 2013 [acesso em 2016 Out 15];18(1):273-80. Disponível em: http://www.scielosp.org/pdf/csc/ v18n1/28.pdf

13. Andrade R. Uma análise do impacto do programa saúde da família sobre a taxa de mortalidade infantil nos municípios do estado de alagoas. Economia Rev [Internet]. 2013 [acesso em 2016 Jun 24];21(2):17-35. Disponível em: http://ojs.uem.br/ ojs/index.php/EconRev/article/view/21346/pdf

14. Mendes EV. 25 anos do Sistema Único de Saúde: resultados e desafios. Estud Av [Internet]. 2013 [acesso em 2016 Set 04];27(78):27-34. Disponível em: http://www.scielo.br/pdf/ea/v27n78/03.pdf

15. Castro ALB, Machado CV. A política federal de atenção básica à saúde no Brasil nos anos 2000. Physis (Rio de J) [Internet]. 2012 [acesso em 2016 Set 28];22(2):477-506. Disponível em: http://www.scielo.br/pdf/physis/v22n2/05.pdf

16. Lima L, Andrade CLT. Condições de financiamento em saúde nos grandes municípios do Brasil. Cad Saúde Pública [Internet]. 2009 [acesso em 2016 Set 28];25(10):2237-48. Disponível em: http://www.scielo.br/pdf/physis/v22n2/05.pdf

17. Rosa MRR, Coelho TCB. O que dizem os gastos com o Programa Saúde da Família em um município da Bahia? Ciênc Saúde Coletiva [Internet]. 2011 [acesso em 2016 Ago 04];16(3):1863-73. Disponível em: http://www.scielo.br/pdf/csc/ v16n3/21.pdf

18. Scatena JHG, Takana OY. A descentralização da saúde no Estado de Mato Grosso, Brasil: financiamento e modelo de atenção. Rev Panam Salud Publica [Internet]. 2000 [acesso em 2016 Set 04];8(4):242-9. Disponível em: http://www. scielosp.org/pdf/rpsp/v8n4/3550.pdf

19. Campos GW. Reforma política e sanitária: a sustentabilidade do sus em questão? Ciênc Saúde Coletiva [Internet]. 2007 [acesso em 2016 Jul 27];12(2):301-6. Disponível em: http://www.scielo.br/pdf/csc/v12n2/a02v12n2.pdf

20. Heimann LS, Ibanhes LC, Boaretto RC, Castro IEN, Telesi E Júnior, Cortizo CT, et al. Atenção primária em saúde: um estudo multidimensional sobre os desafios e potencialidades na Região Metropolitana de São Paulo (SP, Brasil). Ciênc Saúde Coletiva [Internet]. 2011 [acesso em 2016 Set 10];16(6):2877-87. Disponível em: http://www.scielo.br/pdf/csc/ v16n6/25.pdf

21. Domingos CM, Nunes EFPA, Carvalho BG, Mendonça FF. A legislação da atenção básica do Sistema Único de Saúde: uma análise documental. Cad Saúde Pública [Internet]. 2016 [acesso em 2016 Set 10];32(3):1-13. Disponível em: http://www. scielo.br/pdf/csp/v32n3/0102-311X-csp-32-03-e00181314.pdf

22. Sousa MF. O Programa Saúde da Família no Brasil: análise do acesso à atenção básica análise do acesso à atenção básica. Rev Bras Enferm [Internet]. 2007[acesso em 2016 Set 12];61(2):153-8. Disponível em: http://www.scielo.br/pdf/reben/ v61n2/a02v61n2.pdf

23. Porto SM,Santos IS, Ugá MAD. A utilização de serviços de saúde por sistema de financiamento. Ciênc Saúde Coletiva [Internet]. 2006 [acesso em 2016 Ago 10];11(4):895-910. Disponível em: http://www.scielo.br/pdf/csc/v11n4/32328.pdf

24. Portela GZ, Ribeiro JM. A sustentabilidade econômico-financeira da Estratégia Saúde da Família em municípios de grande porte. Ciênc Saúde Coletiva [Internet]. 2011 [acesso em 2016 Ago 10];16(3):1719-32. Disponível em: http://www.scielo. br/pdf/csc/v16n3/09.pdf 
25. Almeida PF, Giovanella, L. Avaliação em Atenção Básica à Saúde no Brasil: mapeamento e análise das pesquisas realizadas e/ou financiadas pelo Ministério da Saúde entre os anos de 2000 e 2006. Cad Saúde Pública [Internet]. 2008 [acesso em 2016 Jul 18];24(8):1727-42. Disponível em: http://www.scielo.br/pdf/csp/v24n8/02.pdf

\section{Endereço para correspondência:}

Ivanise Brito da Silva

Avenida Prof. Moraes Rego, 1235

Bairro: Cidade Universitária

CEP: 50670-901 - Recife - PE - Brasil

E-mail: ivanisebrito1@gmail.com 